\title{
Fallopian tube insertion into the uterine cavity discovered accidentally during laparoscopic retrieval of a misplaced coil from the pelvic cavity
}

\author{
Costas Panayotidis • Jean-Michel Foidart • \\ Michelle Nisolle
}

Received: 4 May 2007 / Accepted: 4 July 2007 / Published online: 15 August 2007

(C) Springer-Verlag 2007

\begin{abstract}
This article presents for the first time in the literature a case of fallopian tube insertion into the uterine cavity discovered accidentally during laparoscopic retrieval of a misplaced coil from the pelvic cavity.
\end{abstract}

Keywords Intra-peritoneal coil $\cdot$ Intrauterine contraceptive device $\cdot$ Uterine perforation $\cdot$ Fallopian tube $\cdot$ Laparoscopy

\section{Introduction}

Uterine perforation during insertion of a contraceptive device (coil) is rare and has been estimated as 1 in 1,000 insertions. However, this percentage may be increased if the insertion is performed immediately after a termination of pregnancy. In these cases, experienced gynaecologists are often involved in order to prevent such complications or misplacement of the coil.

\section{Case study}

A 22-year-old woman had a surgical (suction-aspiration) termination of a 9-week pregnancy. She opted for an

C. Panayotidis $(\bowtie)$

Department of Obstetrics and Gynaecology,

Manchester University Hospital,

Manchester, UK

e-mail: costapan@hotmail.com

J.-M. Foidart $\cdot$ M. Nisolle

Department of Gynaecology and Obstetrics,

Centre Hospitalier Regional de Citadelle, University of Liege,

Boulevard du 12me De Ligne 1,

4000 Liege, Belgium intrauterine contraception device which was inserted at the end of the operation.

However, no suspicion of any perforation or complication was noted during the cervical dilatation and performance of the termination, and the coil was inserted easily into the cavity. Four days later the patient was admitted to the accident and emergency department with intense abdominal colicky pains. An ultrasound scan confirmed that the uterine cavity was empty and an abdominal X-ray detected the coil in the abdominal cavity (Fig. 1). The patient had antibiotic cover and laparoscopy was indicated in order to retrieve the coil from the abdominal cavity. The diagnostic laparoscopy located the coil embedded in a fibrin pseudo sac between a loop of bowel and the left fallopian tube. The coil was gently removed without bowel trauma (Fig. 2a). The left fallopian tube was found to be adherent to the posterior wall of the uterus. But with a closer look, the distal part of the tube was "absent", and the tube was in continuation with the posterior uterine wall (Fig. 2b). The fallopian tube was carefully grasped and gently separated from the uterus, confirming a $1.5 \mathrm{~cm}$ hole on the left posterior uterine wall (Fig. 2c). Approximately 4-5 $\mathrm{cm}$ of the tube was removed from the uterine posterior hole. The tube had an inflammatory and dilated aspect giving a pseudo aspect of an ectopic pregnancy (Fig. 2c). The pelvic cavity was washed and cleaned. The ovaries and the controlateral tube were normal. Neither mullerian malformation was noted nor were PID and endometriosis seen. Haemostasis was adequate. Antibiotic cover (with Augmentin $875 \mathrm{mg}, 2 \mathrm{X}$ daily for 5 days) was administrated. The postoperative recovery was uneventful and the postoperative $\beta$ hcg level was decreased excluding the coexistence of a heterotopic pregnancy. Further gynaecological appointment was organised in order to discuss the findings and future contraception need. 


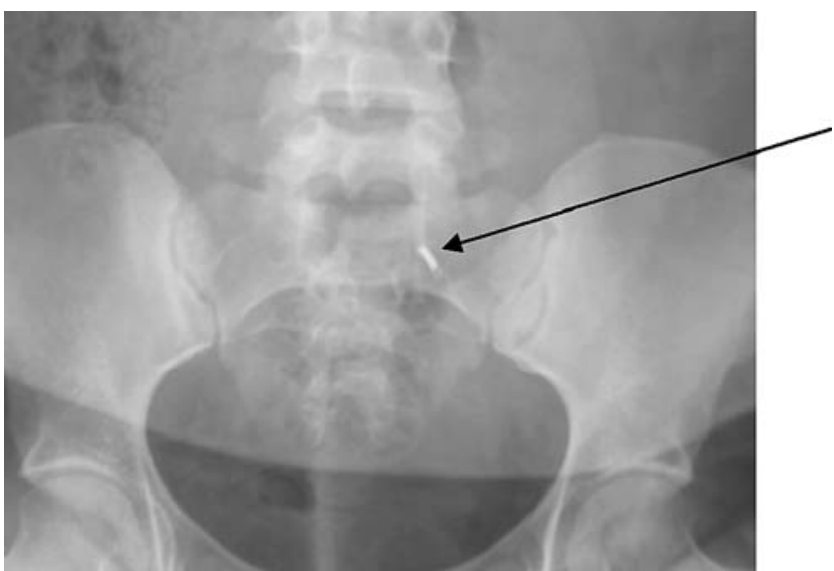

Fig. 1 Abdominal X-ray, arrow pointing to the coil at the left side of the pelvis

\section{Discussion}

The perforation of the posterior wall explains the misplacement of the coil. However, it is difficult to explain when this perforation occurred and how the fallopian tube was inserted into the uterus. The most plausible explanation is that the uterine perforation occurred during the evacuation of retained products of conception and that at this moment the fallopian tube was aspirated into the uterine cavity. The coil probably passed directly to the abdominal cavity through the perforation without returning the tube back into the pelvis. The pressure difference between pelvis and uterine cavity may explain possible adherence between the nearest organ (in our case the fallopian tube) and the uterine posterior wall, but this could not explain how 4$5 \mathrm{~cm}$ of the fallopian tube was introduced into the uterine cavity. Few reports of aspirated bowel with
Fig. 2 Laparoscopic view. a Coil retrieval. b Fallopian tube into the uterus. c Posterior site of uterine perforation (hole) and fallopian tube pseudo aspect of ectopic
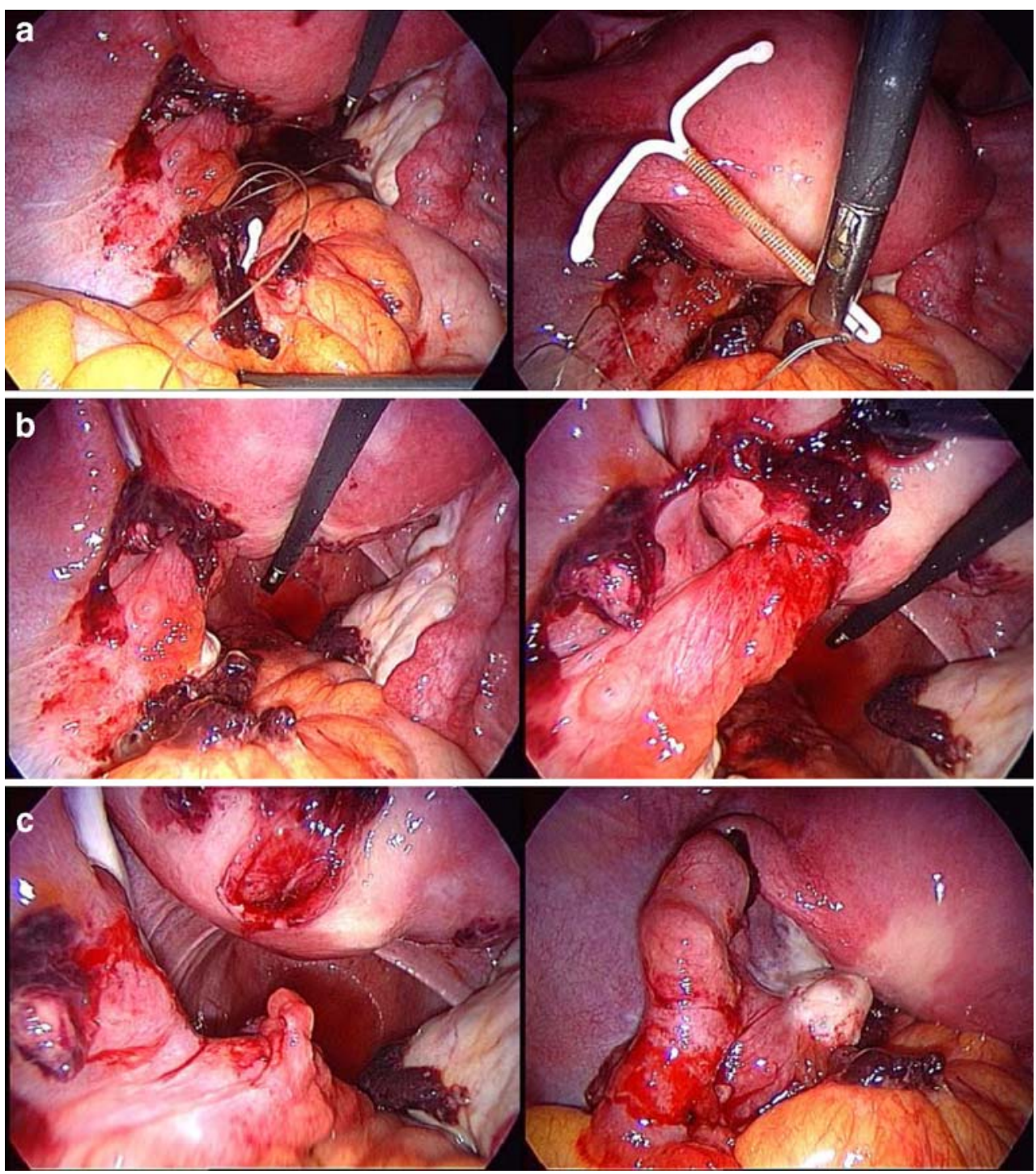
suction-evacuation during termination of pregnancy have been reported and none involve the fallopian tube. There are serious risks of bowel perforation or pelvic abscess if bowel is involved [1-3]. In cases of misplaced coil into the abdominal cavity without bowel perforation, the patient risks pregnancy, pelvic infection, adhesion formation, and chronic pain [4]. The inflammatory aspect of the fallopian tube could be explained by its abnormal placement in the uterine cavity and by mild ischemia due to the mechanical compression from myometrium. For our patient, prompt diagnosis and management was successful. Most of the authors agree that removal of intraperitoneal coil is necessary as it provokes local inflammation and adhesion formation [5-7]. In our case, fibrin deposits had already formed around the coil, which was surprisingly early considering that only 4 days had past since its insertion. The laparoscopic location of the coil can be tricky if there is no sign of adhesions [8]. An abdominal $\mathrm{X}$-ray can point out the side where the coil is located. During laparoscopy, careful bowel manoeuvres should be undertaken in order to detect the coil. Laparoscopic retrieval of the missed coil is considered the standard approach, and laparotomy is performed very rarely $[7,9$, 10]. Finally, fallopian tube insertion into the uterine cavity discovered accidentally during laparoscopy is a very rare clinical scenario. Even though a misplaced coil in the abdominal cavity can be easily retrieved by laparoscopy, the need for thorough inspection of all the organs should be mandatory. In our case, "just removing the coil" without systematic inspection of the pelvic organs could miss such a rare complication with future adverse effect on the patient's fertility.

\section{References}

1. Mederos R, Humaran L, Minervini D (2007) Surgical removal of an intrauterine device perforating the sigmoid colon: a case report. Int J Surg (in press)

2. Hogston P (1986) Removal of an intra-uterine contraceptive device per rectum. J Obstet Gynaecol 7(1):75

3. Jatti J, James S, James MC (2007) Peritoneal abscess resulting from a translocated copper intrauterine device. J Fam Plann Reprod Health Care 33(1):57-58

4. Fuller RR, Roddenberry E (2006) Pelvic pain associated with an unsuspected intraomental intrauterine device placed 30 years earlier: a case report. J Reprod Med 51(9):753-756

5. Mittal S, Gupta I, Lata P, Mahajan U, Gupta AN (1986) Management of translocated and incarcerated inatruterine contraceptive devices. Aust N Z J Obstet Gynaecol 26(3):232-234

6. Ozgun MT, Batukan C, Serin IS, Ozcelik B, Basbug M, Dolanbay M (2007) Surgical management of intra-abdominal mislocated intrauterine devices. Contraception 75(2):96-100

7. Cristinelli S, Nisolle M, Foidart JM (2006) Uterine perforation by an intrauterine device. Rev Med Liege 61(11):747-749

8. Mulayim B, Mulayim S, Celik NY (2006) A lost intrauterine device. Guess where we found it and how it happened. Eur J Contracept Reprod Health Care 11(1):47-49

9. McKenna PJ, Mylotte MJ (1982) Laparoscopic removal of translocated intrauterine contraceptives devices. $\mathrm{Br} \mathrm{J}$ Obstet Gynaecol 89(2):163-165

10. Miranda L, Settembre A, Capasso P, Cuccurullo D, Pisaniello D, Corcione F (2003) Laparoscopic removal of an intraperitoneal translocated intrauterine contraceptive device. Eur J Contracept Reprod Health Care 8(2):122-125 